\title{
Determination of Wound Healing Activity of Shark Liver Oil using Burn Wound Model in Wistar Rats
}

\author{
Samiullah Allahbaksh Auti ${ }^{1, *}$, Rakesh Kumar Jat ${ }^{1}$, Supriya Kumar Das ${ }^{2}$, ST Shukla ${ }^{3, *}$ \\ 'Pharmaceutical Sciences, Shri JJT University, Jhunjunu, Rajasthan, INDIA. \\ 2 Pretox Research Centre, Sachin, Surat, Gujarat, INDIA. \\ ${ }^{3}$ Drug Discovery and Development Division, Patanjali Research Institute, Haridwar, Uttarakhand, INDIA.
}

\begin{abstract}
Background: Research on wounds has proved that a wound instigates interruption to skin integrity caused by physical trauma or disease. Burn is a wound which causes tissue coagulant necrosis and thus exerts a devastating effect on individuals in terms of human health and injury. The present study is designed to evaluate wound healing properties of Shark liver oil and estimate various parameters such as, SOD, CAT, GSH, hydroxyproline and histopathological studies. Methods: Thermal injuries were done with a solid aluminum bar $10 \mathrm{~mm}$ in diameter formerly heated in boiling water to facilitate the temperature reached $100^{\circ} \mathrm{C}$ measured with a thermometer. Ketamine at a dose of $80 \mathrm{mg} / \mathrm{kg}$ is given to produce anesthesia and the hair on dorsum of rats were shaved to ensure even burn wounding. From day- 1 to end of experiment (30 days), these rats were applied with Standard (Silver Sulphadiazine Ointment USP 5\% $\mathrm{w} / \mathrm{W}$ ) and SLO (Shark liver oil emulgel, 5\%, 10\% and 15\%) formulations were applied $1 \mathrm{mg} / \mathrm{cm}^{2}$; topically. Animals were subsequently examined for biochemical and histopathological variations. Results: In burn wound model the developed shark liver oil emulgel shows significant wound healing property in dose dependent manner as compare to disease control
\end{abstract}

groups. The wound healing property of shark liver oil emulgel $5 \%, 10 \%$ and $15 \%\left({ }^{*} p<0.05, * * 0<0.01\right)$ showed wound healing on $3^{\text {rd }}$ and $4^{\text {th }}$ week respectively. Conclusion: The shark liver oil emulgel exhibited significant wound healing activity in burn wound model, comparable to the marketed standard formulations. This finding provides an insight into the usage of the shark liver oil emulgel in the treatment of wounds or burns.

Key words: Burn wound, Percentage wound closure, Hydroxyproline, Epithelization, Shark liver oil and Wound healing activity.

Correspondence

Mr. Samiullah Allahbaksh Auti

Pharmaceutical Sciences, Shri JJT University, Jhunjunu, Rajasthan, INDIA. Email: samidon25@gmail.com

Mr. Sunil T Shukla

Patanjali Research Institute, Haridwar, Uttarakhand-249405, INDIA.

Email: sunilshukla2484@gmail.com

DOI: 10.5530/jyp.2021.13.24

\section{INTRODUCTION}

Wounds inflict mild to significantly injurious damage to a natural tissue structure that results in a wide variety of molecular and cellular alterations. ${ }^{1}$ Wound healing is a mechanism involving inflammation, epithelization, granulated tissue formation, and tissue reshaping process of collagen synthesis. ${ }^{2}$ Anti-inflammatory drugs, corticosteroids, and anti-cancer medicines are some of the causes that reverse and delayed wound remedies. ${ }^{3,4}$ Various animal models are used in wound-healing research. Rat models are the ones mainly used, probably because of preexisting sophisticated research methodologies as the appropriate care and ethical utilization of these species are highly developed and organized to serve science throughout the world. Burn is a wound that produces tissue coagulant necrosis wields a harmful effect on individuals. Skin wounds and conciliated wound healing are major apprehensions for the public health sector. Composite and extended treatments cause an increasing burden on healthcare disbursements. Even in uncomplicated cases, burns and chronic wounds require surgery and extended hospitalization periods. ${ }^{5}$ Burns are one of the most common and shocking forms of trauma. Moderate to severe burn injuries requires hospitalization and many patients die from burn-related complications. ${ }^{6}$ The massive health benefits of the shark liver oil have been deliberated in the past few decades and were described as a source of nutrients such as lipid-soluble vitamins and essential fatty acids. ${ }^{7}$
Because of its therapeutic health advantages such as wound healing property and the motivation of hematopoiesis, it is being used in traditional medicine. Shark liver oil is known as natural amazing healer. ${ }^{8}$ The physiochemical parameters screening of shark liver oil shows that it has amazing healing properties, due to the presence of major active constituents like Squalene. ${ }^{9}$ Research on animals shows that Shark liver oil shield against chemically induced skin, colon, and lung cancer owing to its antioxidant capacity, which combats the cancer-inducing effects of free radicals, as well as by intervening with the cancer-causing influence of certain molecules. ${ }^{10,11}$ SLO (Shark liver oil emulgel, 5\%, $10 \%$ and $15 \%$ ) supplemented diets given to rats with ischemic heart disease and hypertension, perk up clinical symptoms, anthropometric levels, lipidemic profile as well as boost immunological status. ${ }^{12}$ SLO is also suggested in patients diagnosed with atopic dermatitis caused by bacterial and fungal infections. ${ }^{13}$ Shark liver oil is endorsed as a dietary supplement used to boost up the immune system, resist infections, heal wounds, and to heal cancer and decrease the side effects of conventional cancer therapy. Alkyl glycerol, one of the constituents found in shark liver oil, are thought to be beneficial in several ways. It has been suggested that they fight cancer by killing tumor cells indirectly. Shark liver oil has been used in the treatment of wound healing, and for treating irritations of the respiratory plus alimentary tracts and lymphadenopathy. ${ }^{14}$ The formulations are prepared on the basis of above-mentioned concepts

This is an open access article distributed under the terms of the Creative Commons Attribution-NonCommercial-ShareAlike 4.0 License, which allows others to remix, tweak, and build upon the work non-commercially, as long as the author is credited and the new creations are licensed under the identical terms. 
and standard principles present in shark liver oil for the purpose of its antioxidant and wound curing properties in the concentration of $(5 \%, 10$ $\%$, and $15 \%$ ) percent respectively.

\section{MATERIALS AND METHODS}

\section{Animals}

Albino Wistar Rats (180-200 g) healthy adults were selected for the test. They have been purchased from Mahaveera Enterprises, Hyderabad, India. The experimental Protocol (No: PRC/AFC/2019/310) was adopted after approval by the Institutional Animal Ethical Committee formed under regulation of CPCSEA at Pretox Research Center, Sachin area, Surat, Gujarat, India. Animals were acclimated over seven days under natural illumination of $12: 12 \mathrm{hrs}$ of light and dark period, $23 \pm 2{ }^{\circ} \mathrm{C}$ of temperature, and humidity of approximately 50 percent in the normal laboratory conditions. For the whole study, the animals have been fed normal diet and water with ad libitum.

\section{Drugs and Chemicals}

Analytical grade chemicals, drugs and standard reagents such as sodium hydroxide ketamine. Silver sulfadiazine, Carbopol 940, etc were procured from M/s S.K. Appliances, Ambala City, Punjab, India with voucher number SKA/2019-20/10025 appropriately. Shark liver oil was procured from Mr. Nissar F.M, Coastal Exports Corporation, Mangalore, India as a gift sample.

\section{Grouping of Animals}

The grouping of the animals in burn wound model was as follow:

Group - 1 Disease Control (DC)

Group -2 Standard Group (STD) Animals were treated with Silver Sulphadiazine Ointment USP 5\% w/w.

Group -3 Shark Liver oil, 5\% Emulgel (SLO 5\%)

Group -4 Shark Liver oil, 10\% Emulgel (SLO 10\%)

Group -5 Shark Liver oil, 15\% Emulgel (SLO 15\%).

\section{Experimental Method for Burn Wound Model}

Primarily albino Wistar rats were procured with weight of 180-200 g and randomized before grouping. The animals were pre-anesthetized with atropine sulfate $(0.04 \mathrm{mg} / \mathrm{kg})$ intramuscularly and $10 \mathrm{~min}$ after subjected to anesthesia using a mixture of $10 \%$ ketamine $(90 \mathrm{mg} / \mathrm{kg})$ and $2 \%$ xylazine $(10 \mathrm{mg} / \mathrm{kg})$ given intramuscularly. With the animal, suitably anesthetized tracheotomy of back was executed, and 1\% polyvinyl iodine applied as antiseptic. Thermal injuries were done with a solid aluminum bar $10 \mathrm{~mm}$ in diameter formerly heated in boiling water to facilitate that the temperature reached $100^{\circ} \mathrm{C}$ measured with a thermometer. The bar is retained in contact with the animal skin on the dorsal proximal region for 15 secs, the pressure put forth on the animal skin match up to the mass of $51 \mathrm{~g}$ of aluminum bar used in the burn induction. Instantly after the procedure, analgesia with dipyrone sodium $(40 \mathrm{mg} / \mathrm{kg}$ ) was given intramuscularly, lasting for three consecutive days, sodium dipyrone at $200 \mathrm{mg} / \mathrm{kg}$ orally dispensed in the drinking water supplied to animals. ${ }^{15}$

\section{Method of Obtaining Shark Liver Oil}

The shark liver oil is obtained from the fresh livers of different shark types: deep-water Centrophorus squamosus sharks. The livers are separated from the sharks, cleaned, and are unbound from fatty matter and adhering tissues. The untainted livers are collected and heated in a boiling vessel at a temperature not exceeding $80^{\circ} \mathrm{C}$. The extracted oil is redirected to dehydration process to purge water and centrifuged to obtain clear oil. Shark liver oil obtained is $100 \%$ pure, devoid of artificial coloring, preservatives, or additives. ${ }^{16}$ Large amounts of constituent like Squalene are found in fully processed shark liver oil. ${ }^{17}$

\section{Formulation of Shark Liver Oil Emulgel}

The formulations in concentrations of $5 \%, 10 \%$, and $15 \%$ of shark liver oil emulgel were used to prepare $100 \mathrm{~g}$ of gel by applying enough purified water to Carbapol-940, propylene-glycol 400, ethanol, propyl-paraben, EDTA, and tri-ethanolamine respectively. The required water was divided into two components for the formulations. One part dissolved the exact volume of shark liver oil and added with propylene glycol 400. Ethanol is added to this mixture and in another one-part dissolved Carbapol-940 added with propyl paraben, and EDTA to this solution. Both solutions have been blended into a beaker and ethanolamine is added to the mixture to create a gel consistency. A homogenous gel without intact air bubbles was collected using a propeller at 500 mins for $2 \mathrm{hrs}^{18}$

\section{Biomarkers and Biochemical Estimations}

After the completion of experiment, the extracted granuloma tissue from the burn wound was washed in phosphate buffered saline $(\mathrm{pH} 7.0)$ and centrifuged under cold conditions. The transparent supernatant was assayed using spectrophotometer and the levels of antioxidant enzymes like SOD, CAT and GSH are determined. ${ }^{19} \mathrm{~A}$ piece of skin from the healed wound area was collected and analyzed for hydroxyproline content, which is basic constituent of collagen. Tissues were dried in a hot air oven at $60-70^{\circ} \mathrm{C}$ to constant weight and were hydrolyzed in $6 \mathrm{~N}$ $\mathrm{HCl}$ at $130^{\circ} \mathrm{C}$ for $4 \mathrm{hrs}$ in sealed tube. ${ }^{20}$

\section{Histopathological Study}

Tissue healing samples from all five animal groups were taken and subjected for histological analysis. The samples were fixed formally and placed on the slides and then examined by an automated illumination microscope, stained by hematoxylin and eosin. Scars, inflammation cells, epidermal hyperplasia, neovascularization, hyperpolarization, fibroblastic aggregation, and ulcer scores were all documented.

\section{Statistical Analysis}

Results are expressed as mean \pm SEM. The differences between experimental groups were compared by one-way Analysis of Variance (ANOVA) (control vs. treatment) followed by Tukey's Multiple Comparisons Test and were considered statistically significant when $\left({ }^{*} p<0.05,{ }^{* *} p<0.01\right) .{ }^{21}$

\section{RESULTS}

It is inferred from the present analysis that there is a substantial decrease in epithelization after using SLO (5\%,10\%, and 15\%) compared to the regular standard drug. Therefore, comparative research has shown that formulations of SLO produces significant wound healing effect.

\section{Relative Bodyweight Changes}

The growth rate of disease control rats was dissimilar before and after the period of burn injury. The injured animals illustrated a response characterized by weight loss from day 0 to day 15. In burned animals treated with standard and SLO have shown rapid loss of fat over 15 days and reached a minimum weight gain by 25 days. This level is sustained for remainder of experiment and have shown significant increase in body weight as shown in Figure 1.

\section{Wound Area}

On days 1, 5, 10, 15, 20, 25 and 30 wound area (in $\mathrm{cm}^{2}$ ) was measured. The wound area of burned rats increased initial 5 days and gradually decreased progressively. The burn wound treated with SLO healed more 
quickly. There is a significant increase in wound healing activity of SLO treated groups in comparison to disease control group. ( $\left.{ }^{* *} P<0.01\right)$ as shown in Figure 2.

\section{Relative Burn Wound Area (\% Percentage)}

The area of wound was measured on the 1, 5, 10, 15, 20, 25, and 30 days of post burn wound surgery in all groups. It has shown significant reduction in relative burn wound area in \% percentage of SLO treated different groups over period of 30 days. The SLO treated group have shown significant decrease in burn wound area post 25 days as compared to disease control groups. ( $\left.{ }^{\star *} P<0.01\right)$ as shown in Figure 3 .

\section{Epithelization Period}

A very high rate of closure of wound, the epithelization was observed after 28 days in treated groups. SLO and standard shown significant wound healing activity $\left({ }^{*} p<0.05,{ }^{* *} p<0.01\right)$ and gradual closure of wound by $20^{\text {th }}$ day of post wound surgery and by 30 days in control groups. Both standard and SLO treated group showed decreased epithelization period as compare to disease control groups as shown in Figure 4.

\section{Antioxidant Activity}

Shark liver oil emulgel has shown major improvement in wound healing process and antioxidant property has shown decreasing SOD and increasing GSH, CAT, and Hydroxyproline concentration in all treated groups. There is significant decrease in SOD level in SLO treated groups as compare to disease control. Also, the results have proved that there is an increased level of GSH, CAT and Hydroxyproline concentrations in

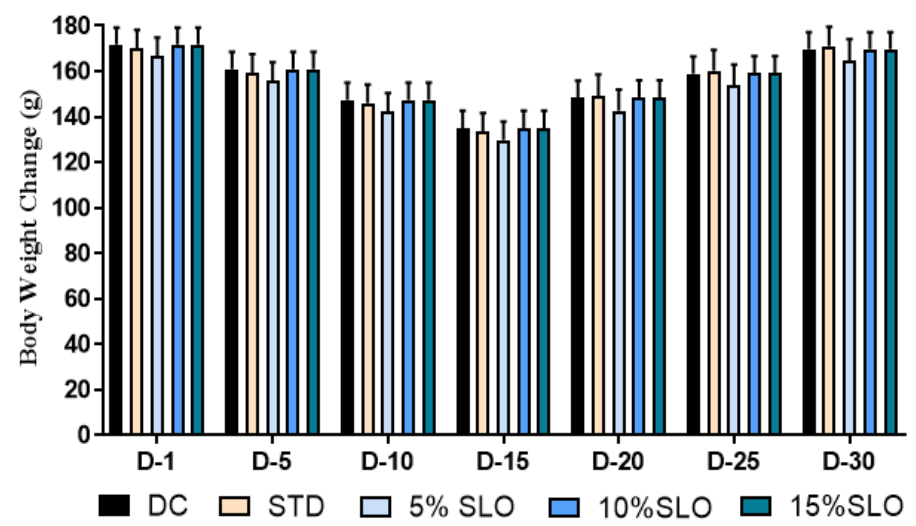

Figure 1: Body weight changes.

All values are represented as Mean \pm SEM, $n=6$, Data was analyzed by Twoway ANOVA, followed by Tukey's Multiple Comparisons Test.

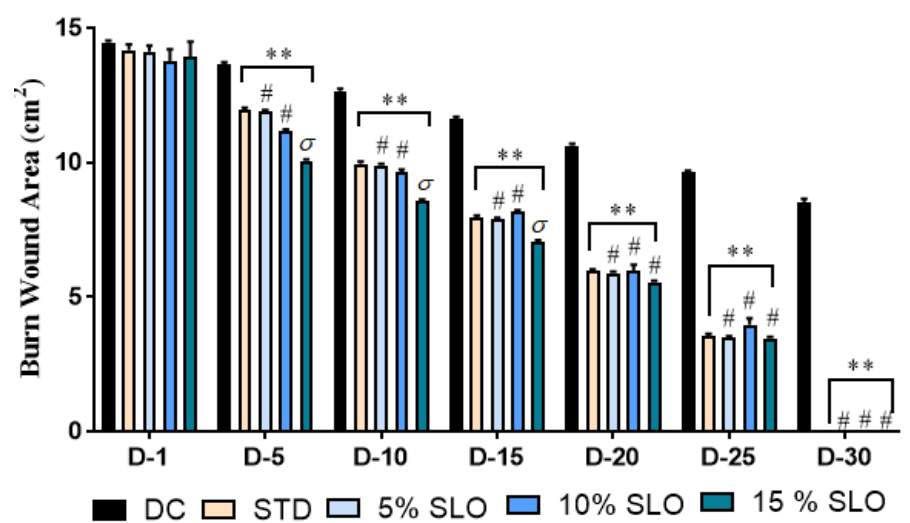

Figure 2: Wound area in $\mathrm{cm}^{2}$.
SLO treated groups. This reveals significant wound healing action of SLO as shown in Figure 5 (A-D).

\section{Histopathology and Microscopic Observation}

Histopathological findings and microscopic examination shows that topically applied SLO has notably improved wound contraction, collagenation, and epithelization with well-organized dermis devoid of inflammatory cells as contrast to disease control. Histological assessment of the hematoxylin stained tissue of the rat wounds treated with SLO and standard has illustrated superior healing in abrasion numbers as shown in histograms. Figure 6 (A-E).

\section{DISCUSSION}

In present research, the percentage of wound contraction and histopathological results have shown relative changes in body weight and substantial improvement in the efficiency of tissue granulation by raising the fibroblast proliferation and elevating the degree of maturation of collagen material, and a major rise in the wound care application of the shark liver oil emulgel. Relative body weight alteration from slow weight loss over the first 15 days to that of gain till 30 days was supposed to be a

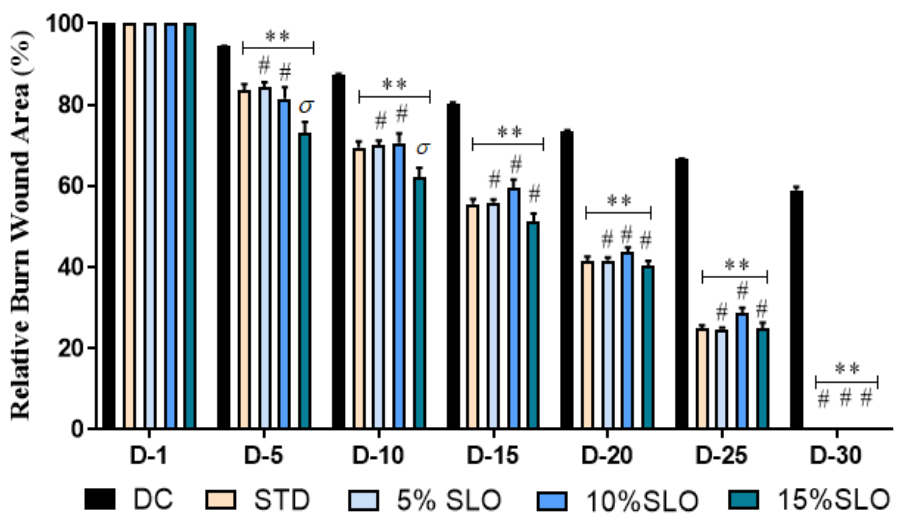

Figure 3: Relative burn wound area (\%).

All values are represented as Mean \pm SEM, $n=6$, Data was analyzed by Twoway ANOVA, followed by Tukey's Multiple Comparisons Test. ${ }^{*} p<0.05$, ${ }^{* *} p<0.01$ significantly different as compared to DC. \#p $>0.05$ non-significant in comparison to Sliver sulfadiazine. $\sigma p<0.05$ significantly different as compared to Sliver sulfadiazine.

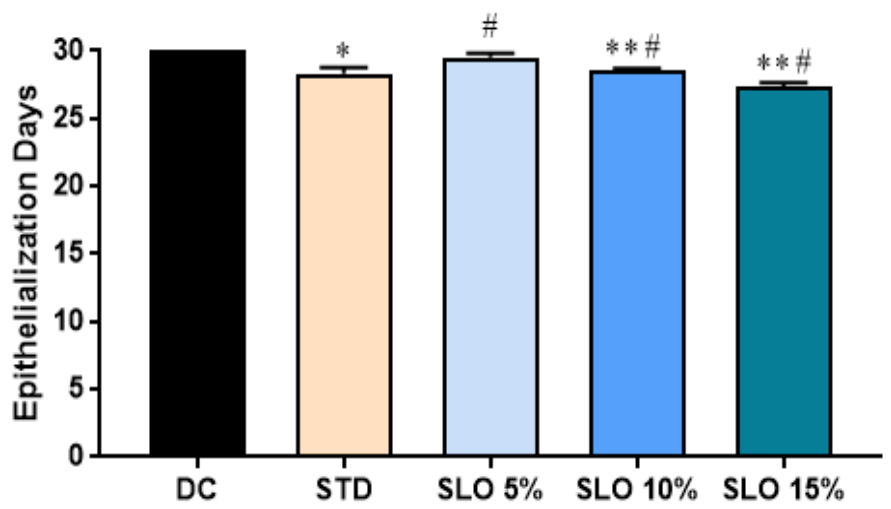

Figure 4: Epithelialization periods.

All values are represented as Mean \pm SEM, $n=6$, Data was analyzed by Oneway ANOVA, followed by Dunnett's Multiple Comparisons Test. * $p<0.05$, ${ }^{* *} p<0.01$ significantly different as compared to DC. $\# p>0.05$ non-significant in comparison to Sliver sulfadiazine. 
straight outcome of marginal wound healing and thus decreases energy requirements. Tissue damage after thermal injury is the outcome of an energy insufficiency brought about by hyper metabolism caused by the decreased food intake instantly after injury. The injured animals in this experiment shows growth retardation and were able to regain their body composition to that of an uninjured animal of the same weight. ${ }^{22}$ It is noticed that there is non-significant increase in relative body weight post 15 days of burn wound Figure 1. The wound healing mechanism chiefly relies on the controlled collagenic fabrication of new collagen deposition and its ensuing maturity. ${ }^{23}$ Collagen is found in profuse quantity in our body. In wound healing, it plays an imperative role by attracting fibroblasts and promotes deposition of new collagen to the wound bed which accelerates new tissue growth and endorses angiogenesis and re-epithelization. ${ }^{24}$ Wound Contraction is a remedial mechanism accomplished by two fundamental proteins actins and myosin depicted by the movement of fibroblasts in granulation tissue collagen and hauls the forces of granulation tissue my fibroblasts on the skin edges Figure 2, Figure $3 .{ }^{25}$ Epithelialization is depicted by movement of epithelial cells in upward direction and renovating the wounded area which predominantly occurs at proliferative phase of wound healing Figure 4. The report demonstrate that, topically applied shark liver oil emulgel $(5 \%, 10 \%$, and $15 \%)$ has established collagenation, wound contraction, epithelialization and has proved its significance in contrast to other fixed oil $\left({ }^{*} p<0.05,{ }^{* *} p<0.01\right) \cdot{ }^{26} \mathrm{GSH}$, amounts of hydroxyprolines, CAT, and SOD are a big mechanism for a variety of damage, such as burned wounds and skin ulcers. Antioxidant medication is thought to improve the viability of collagen fibrils by enhancing the strength of collagen fibers. This facilitates DNA synthesis by avoiding damage to the cells.

Superoxide Dismutase (SOD) an endogenous antioxidant, are a cluster of enzymes that catalyze the dismutation of superoxide radicals $\left(\mathrm{O}_{2-}\right)$ to molecular oxygen $\left(\mathrm{O}_{2}\right)$ and hydrogen peroxide $\left(\mathrm{H}_{2} \mathrm{O}_{2}\right)$ by imparting cellular defense against ROS (reactive oxygen species). ${ }^{27}$ Reduced glutathione (GSH) acts as valuable antioxidant and protects the cellular components from oxidative injury caused by ROS (Reactive Oxygen Species). ${ }^{28}$ Catalases (CAT) are regular enzyme found in all living organisms which when depicted to oxygen catalyzes the breakdown of Hydrogen peroxide to water and oxygen. As a result, the cells are safeguarded from oxidative damage produced by Reactive Oxygen Species (ROS). ${ }^{29}$

Hydroxyproline is main component of protein collagen and plays an important role by providing stability in collagen formation and specify the amount and concentration of collagen in wound healing process. ${ }^{30}$ Higher concentrations of hydroxyprolines indicates rapid rates of wound healing Figures 5 (A-D).
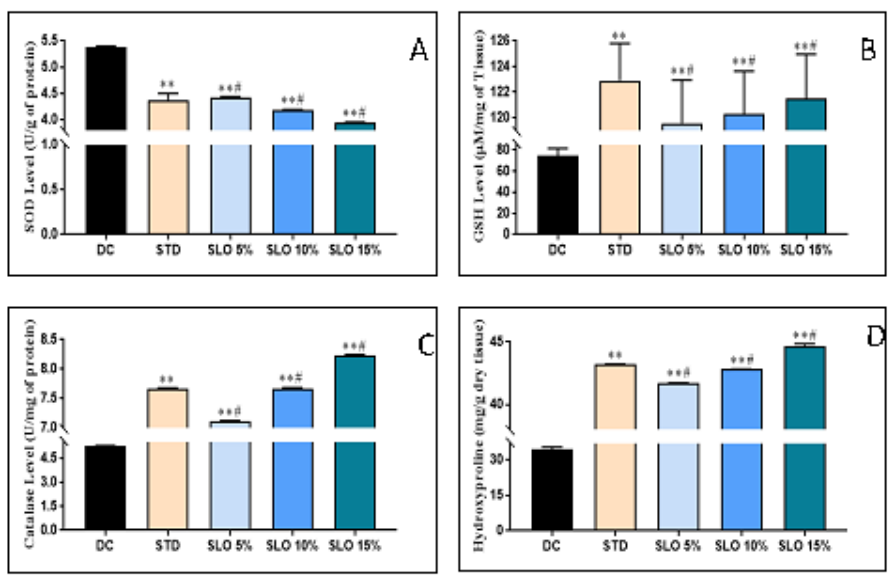

Figure 5 (A-D): Antioxidant activity.

A. SOD Level; B. GSH Level; C. Catalase Level; D. Hydroxproline Level.
The research on skin irritations has shown the absence of a serious type of irritation in the formulation of shark liver oil emulgel when spread over tropically on the skin. ${ }^{31}$ This specifies that the drug may include some chemical components which do not cause any extreme type of irritation. Silver sulfadiazine is a topical antibiotic used in and burns to prevent infections. It acts by distracting bacteria by damaging the cell crust and the cell wall rather than by reducing folic acid synthesis. It has a broad spectrum of bactericidal action against both gram-positive and gram-negative organisms. ${ }^{32}$ Histological measurement of the wound articulates the importance of histopathology. Histopathology evaluation mostly compares diseased or experimentally altered tissues with identical sample from powerful control complements. Therefore, it is very important to thoroughly standardize every histology method. ${ }^{33}$

Histopathology study is characterized by Fibroblast proliferation, Neovascularization, Epidermal Hyperplasia, Inflammatory score, and Ulcer formation. Fibroblast promulgation comprises fibroblasts which are mainly important in the progression of tissue repair. They produce structural proteins, adhesive proteins, and space-filling ground materials necessary in building extracellular matrix of connective tissue. ${ }^{34}$

Neovascularization is also identified as new blood vessel creation, is very prominent in successful tissue refurbishment during wound repair. The novel blood vessels formed supports in supplying oxygen and nutrients from blood to dented tissue. ${ }^{35}$

Epidermal hyperplasia is significant response of connective tissue in which the multiplying fibroblasts and blood vessels aid in wound repair. Inflammatory responses is exemplified by pathogens, damaged cells, and elimination of bacteria in which growth factors, enzymes, White Blood Cells (WBC), and nutrients initiate redness, pain, and bulge. ${ }^{36}$ Ulcers owing to arterial insufficiency often instigate as minor traumatic wounds that remain ineffective to heal. The main tactic to nurture arterial insufficiency ulcers is to restore accurate blood flow to the tissue to develop appropriate wound healing.

Histopathology and histological scoring technique has confirmed the effectiveness of SLO administration in development of the damaging effects on Burn wound healing includes semi-quantitative scoring of inflammatory phase, the amount of granulation tissue, level of reepithelialization, amount of collagen deposition, intensity of Fibroblast proliferation, Neovascularization, Hyperplasia and Ulceration. The
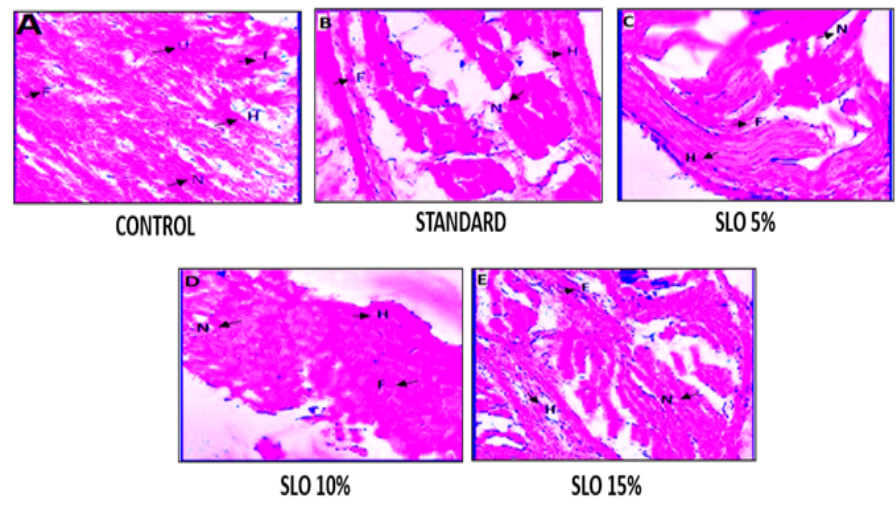

Figure 6 (A-E): Histopathology results.

A. Control - Skin: Showing ulceration (U) inflammation (I) fibrous tissue proliferation $(\mathrm{F})$ and $(\mathrm{N})$ neovascularization in dermis $\{100 \mathrm{X}\}$ B. Standard - Skin: Showing epidermal hyperplasia (H) fibrous tissue proliferation $(\mathrm{F})$ and neovascularization $(\mathrm{N})$ in dermis $\{100 \mathrm{X}\}$ C. SLO-5\% - Skin: Showing epidermal hyperplasia $(\mathrm{H})$ fibrous tissue proliferation ( $\mathrm{F}$ ) and neovascularization $(\mathrm{N})$ in dermis $\{100 \mathrm{X}\}$ D. SLO-10\%: Showing epidermal hyperplasia $(\mathrm{H})$ fibrous tissue proliferation (F) and neovascularization in dermis $\{100 X\}$ E. SLO-15\% - Skin: Showing epidermal hyperplasia (H) fibrous tissue proliferation $(\mathrm{F})$ and neovascularization $(\mathrm{N})$ in dermis $\{100 \mathrm{X}\}$ 
total healing score designated that lower scores verified inferior wound healing in Control groups in contrast to significant healing score in SLO treated groups Figures 6 (A-E).

The burn wound model revealed substantial reductions in epithelization time of animals treated with the SLO formulation, as shown by the shorter duration of eschar reduction relative to stock. The medication also greatly promoted the rate of wound contraction at various stages. Phytochemical tests have shown that SLO contains significant amounts of Squalene and Alcoxyglycerols, an active compound which can induce epithelization in wound healing. The histopathological experiment demonstrated a higher collagen deposition comparison to placebo in the treatment and treated groups. The findings of the present study demonstrate therefore that shark liver oil emulgel topical treatment has increased wound healing in laboratory rats of the burn wound model.

\section{CONCLUSION}

In this analysis, the results have shown that the shark liver oil emulgel has active ingredients that can promote natural treatment and can be used as a wound cure agent effectively. Shark liver oil emulgel increases the solidity of the wound and the epithelialization rate and longevity of the collagen throughout the surface of the wound. The concentrationrelated healing effect in laboratory rats was seen in all three formulations. Further research is required to explore wound healing properties and mechanism of action of the test drug.

\section{ACKNOWLEDGEMENT}

Authors are thankful to Shri JJT University, Jhunjhunu, Rajasthan, for providing necessary facilities and for their support to carry out this Research Project. We are also thankful to Mr. Nissar F.M from Coastal Exports Corporation, Mangalore, Karnataka, for providing the test sample of shark liver oil as a gift to carry out the research work.

\section{CONFLICT OF INTEREST}

The authors declare no conflict of interest.

\section{ABBREVIATIONS}

SLO: Shark Liver Oil Emulgel; SOD: Superoxide Dismutase; GSH: Reduced Glutathione; CAT: Catalases; ROS: Reactive Oxygen Species; DC: Disease Control; STD: Standard Drug; CPCSEA: Committee for the purpose of control and supervision of experiments on animals. PRC: Pretox Research Centre; AFC: Animal Facilitation Centre; hrs: Hours; Secs: Seconds; ROS: Reactive Oxygen Species; SEM: Standard Error Mean; ANOVA: Analysis of Variance.

\section{REFERENCES}

1. Cohen K, Diegelmann R, Lindblad W. Wound Healing; Biochemical Clinical Aspects. W B Sounders, Philadelphia. 1992.

2. Brown GL, Curtsinger LJ, White MI, Mitchell RO, Pietsch JA, Nordquist RO, et al. Acceleration of tensile strength of incisions treated with EGF and TGF-beta. Ann Surg. 1988;208(6):788-94.

3. Stojadinovic O, Lee B, Vouthounis C, Vukelic S, Pastar I, Blumenberg M, et al. Novel genomic effects of glucocorticoids in epidermal keratinocytes: Inhibition of apoptosis, interferon- $\gamma$ pathway, and wound healing along with promotion of terminal differentiation. J Biol Chem. 2007;282(6):4021-34

4. Sen CK, Gordillo GM, Roy S, Kirsner R, Lambert L, Hunt TK, et al. Human skin wounds: A major and snowballing threat to public health and the economy. Wound Repair Regen. 2009;17(6):763-71.

5. American Burn Association. Burn incidence and treatment in the US National health interview survey (1991-1993 data). American Burn Association, Philadelphia, Pa. 2000.

6. Deniau AL, Mosset P, Pédrono F, Mitre R, LeBot D, Legrand AB. Multiple beneficial health effects of natural alkylglycerols from shark liver oil. Mar Drugs 2010;8(7):2175-84

7. Solomon N, Passwater R, Joelssun I, Haimes L. Shark liver oil; Nature's Amazing Healer. Kensington Books. 1997.

8. Pugliese PT, Jordan K, Cederberg H, Brohult J. Some biological actions of alkylglycerols from shark liver oil. J Altern Complement Med. 1998;4(1):87-99.

9. Davidson BC, Rotenberg D, Prinz W, Cliff G. The influence of shark liver oil son normal and transformed mammalian cells in culture. In vivo. 2007;21(2):333-7.

10. Iannitti T, Palmieri B. An update on the therapeutic role of alkylglycerols. Marine Drugs. 2010;8(8):2267-300.

11. Nowicki R, Barańska-Rybak W. Shark liver oil as a supporting therapy in atopic dermatitis. Pol Merkur Lekarski. 2007;22(130):312-3.

12. Palmieri $B$, Pennelli $A$, DiCerbo $A$. Jurassic surgery and immunity enhancement by alkyglycerols of shark liver oil. Lipids Health Dis. 2014;13(1):1-5.

13. Nayak BS, Mohan K. Influence of ethanolic extract of Jasminum grandflorum Linn flower on wound healing activity in rats. Indian J Physiol Pharmacol. 2007;51(2):189-94.

14. Bañón R, Piñeiro C, Casas M. Biological observations on the gulper shark Centrophorus granulosus (Chondrichthyes: Centrophoridae) off the coast of Galicia (north-western Spain, eastern Atlantic). Marine Biological Association of the United Kingdom. J Mar Biol Assoc UK. 2008;88(2):411-4.

15. Tsujimoto M, Toyama $Y$. Ueber die unverseifbaren Bestandteile (hoheren Alkohole) der Haifishund Rochen-leberole. I. Chemische Umschau auf dem Gebiet der Fette, Oele, Wachse und Harze. 1922;29:43-5.

16. Avinash S, Gowda DV, Suresh J, Aravind RAS, Srivastava A, Osmani RAM Formulation and evaluation of topical gel using Eupatorium glandulosum Michx. for wound healing activity. Pharm Lett. 2016;8(9):255-66.

17. Aebi HE. Methods in Enzymatic Analysis, Vol 3, $3^{\text {rd }}$ ed, NewYork, Academic Press, Verlag Chemie, Weinheim; 1973;273-86.

18. Gurung S, Basnet NS. Wound healing properties of Carica papaya Latex: In vivo evaluation in mice burn model. J Ethnopharmacol. 2009;121(2):338-41.

19. Armitage P, Berry G, Matthews J. Statistical methods in Medical Research, $4^{\text {th }}$ ed. Oxford: Blackwell Science; 2002.

20. Jacquier M, Crauste $F$, Soulage $C O$, Soula HA. A predictive model of the dynamics of body weight and food intake in rats submitted to caloric restrictions. PLoS One. 2014;9(6):e100073

21. Puratchikody A, Devi CN, Nagalakshmi G. Wound healing activity of Cyperus rotundus Linn. Indian J Pharm Sci. 2006;68(1):97.

22. Baum CL, Arpey CJ. Normal cutaneous wound healing: Clinical correlation with cellular and molecular events. Dermatol Surg. 2005;31(6):674-86.

23. Gabbiani G. The myofibroblast in wound healing and fibro contractive diseases Pathol. 2003;200(4):500-3.

24. Manzuoerh R, Farahpour MR, Oryan A, Sonboli A. Effectiveness of topical administration of Anethum graveolens essential oil on MRSA-infected wounds. Biomed Pharmacother. 2019;109:1650-8.

25. Fridovich I. Superoxide radical and superoxide dismutases. Annu Rev Biochem 1995;64(1):97-112.

26. Pompella A, Visvikis $A$, Paolicchi $A$, DeTata $V$, Casini AF. The changing faces of glutathione, a cellular protagonist. Biochem Pharmacol. 2003;66(8):1499-503.

27. Biosmenu D, Lépine F, Gagnon M, Dugas $H$. Catalase activity measurement with the disk flotation method. Anal Biochem. 1989;178(2):404-7.

28. McAnulty RJ. Methods for measuring hydroxyproline and estimating in vivo rates of collagen synthesis and degradation. Methods Mol Med. 2005;117:189207

29. Gfeller W, Kobel W, Seifert G. Overview of animal test methods from skin irritation. Food Chem Toxicol. 1985;23(2):165-8.

30. Baxter CR. Topical use of $1.0 \%$ silver sulfadiazine, Contemporary burn management. Little, Brown and Co., Boston. ;217-25.

31. Winsor L. Tissue processing. Laboratory Histopathology: A Complete Reference. New York: Churchill Livingstone; 1994.

32. Reddy GK, Stehno-Bittel L, Enwemeka CS. Laser photostimulation accelerates wound healing in diabetic rats. Wound Repair Regen. 2001;9(3):248-55.

33. Kalka C, Masuda H, Takahashi T, Kalka-Moll WM, Silver M, Kearney M, et al. Transplantation of ex vivo expanded endothelial progenitor cells for therapeutic neovascularization. Proc Natl Acad Sci. 2000;97(7):3422-7.

34. Li J, Chen J, Kirsner R. Pathophysiology of acute wound healing. Clin Dermatol. 2007;25(1):9-18

35. Ortonne JP, Clévy JP. Physiology of cutaneous cicatrization. Rev Prat 1994;44(13):1733-7.

36. Abramov Y, Golden B, Sullivan M, Botros SM, Miller JJ, Alshahrour A, et al. Histologic characterization of vaginal vs. abdominal surgical wound healing in a rabbit model. Wound Repair Regen. 2007;15(1):80-6. 